1

2

\section{Supporting Information}

\section{Contents}

10 Additional NMR Data

Synthetic Optimisation Characterisation Data.

Washing and Devices

\section{Common Abbreviations}

Oleic Acid - OA

Trioctylphosphine Oxide - TOPO

Full width at half maximum - FWHM

Photoluminescence (quantum yield) - PL(QY)

Deuterated Toluene - d-toluene

Cesium oleate - Cs-oleate

Formamidinium oleate - FA-oleate

\title{
A phosphine oxide route to formamidinium lead
}

\section{tribromide nanoparticles}

Olivia J. Ashton, Ashley R. Marshall, Jonathan H. Warby, Bernard Wenger*, Henry J.

Snaith*

Clarendon Laboratory, Department of Physics, University of Oxford, Parks Road, OX1 3PU,

Oxford, UK 


\section{Additional NMR Data}

27

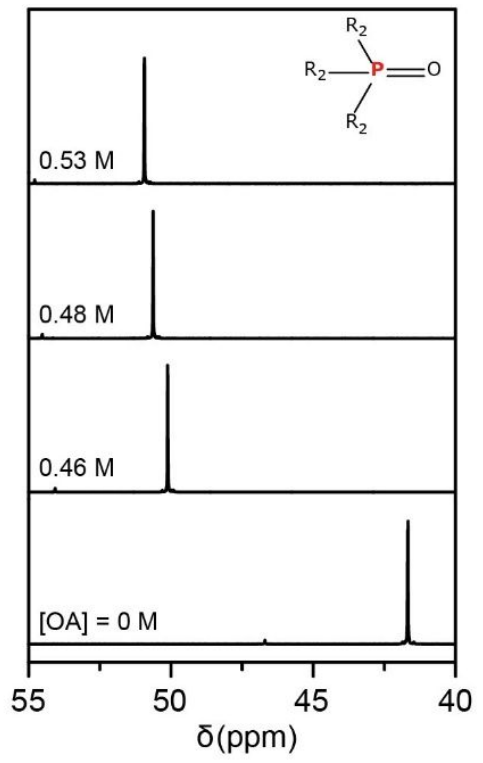

Figure S1 ${ }^{31} \mathrm{P}$ NMR spectra of TOPO P (highlighted in red, $\left.\mathrm{R}_{2}=\mathrm{CH}_{3}\left(\mathrm{CH}_{2}\right)_{7}\right)$ in OA:TOPO solutions, with TOPO fixed at

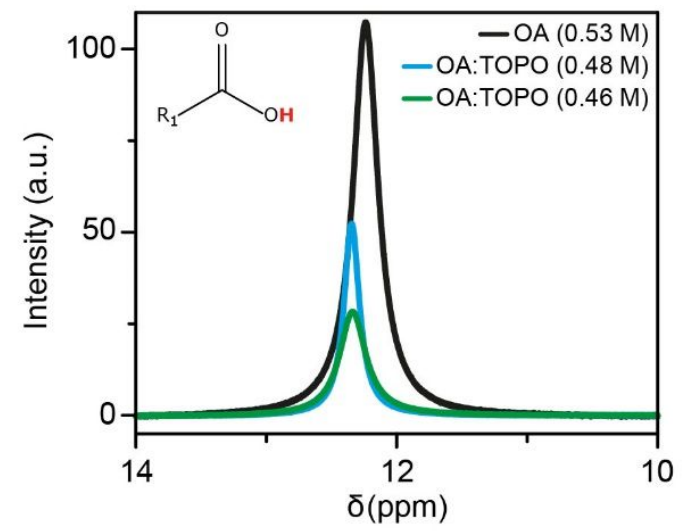

Figure $\mathbf{S 2}{ }^{1} \mathrm{H}$ NMR spectra of the region for the acidic proton on $\mathrm{OA}$ (highlighted in red, $\left.\mathrm{R}_{1}=\mathrm{CH}_{3}\left(\mathrm{CH}_{2}\right)_{7} \mathrm{CH}=\mathrm{CH}\left(\mathrm{CH}_{2}\right)_{7}\right)$ in a solution of OA in d-toluene ( $0.53 \mathrm{M}$, black), and OA:TOPO in d-toluene ([TOPO] $=0.43 \mathrm{M})$ at $0.48 \mathrm{M}$ (blue), and $0.46 \mathrm{M}$ (green) 
a)

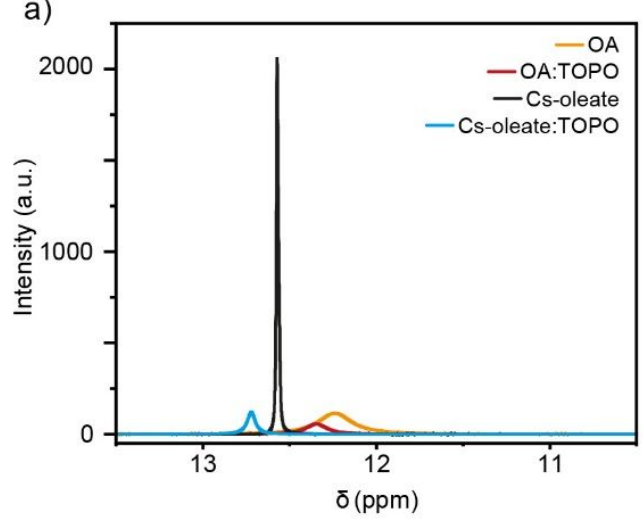

b)

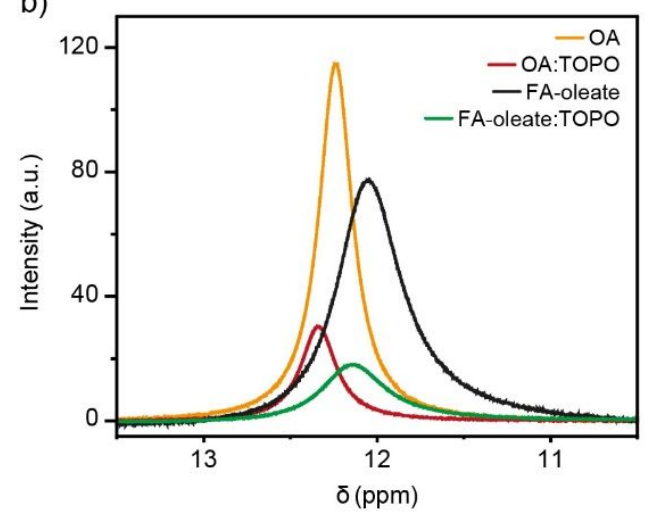

3
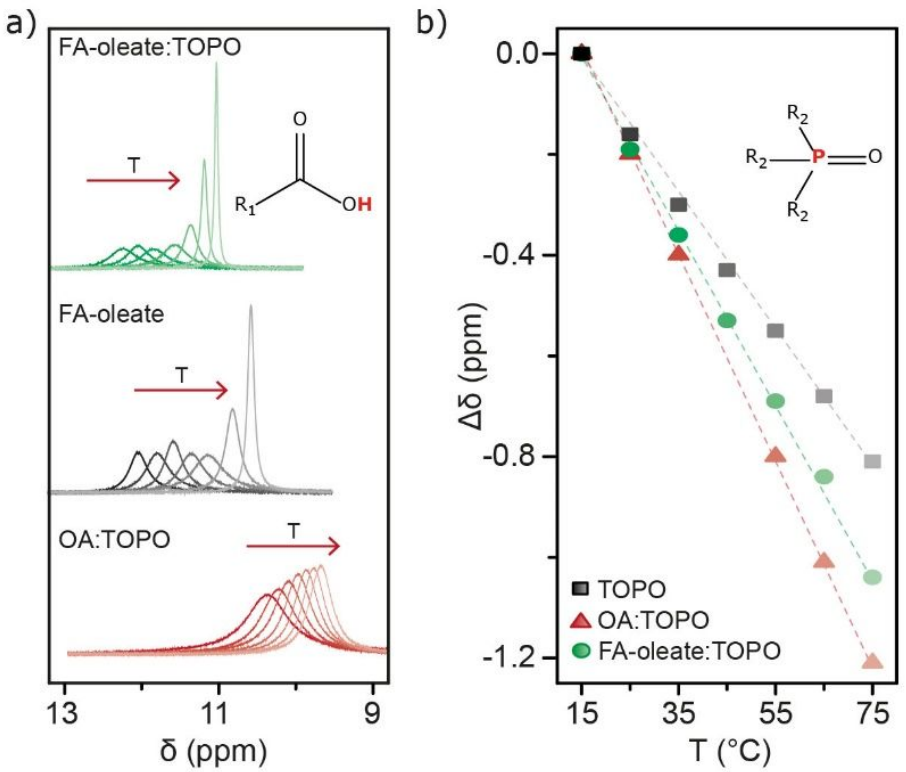

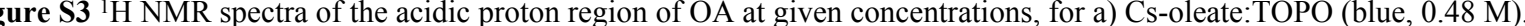
Cs-oleate (black, $0.48 \mathrm{M}$ ), OA:TOPO (red, $0.48 \mathrm{M}$ ) and OA (yellow, 0.53 M) and b) FA-oleate:TOPO (green, $0.46 \mathrm{M}$ ), FAoleate (black, $0.46 \mathrm{M}$ ), OA:TOPO (red, $0.46 \mathrm{M}$ ) and OA (yellow, $0.53 \mathrm{M}$ ). In all cases, the TOPO concentration is $0.43 \mathrm{M}$

Figure S4 a) ${ }^{1} \mathrm{H}$ NMR spectra of the region for the acidic proton on OA (highlighted in red, $\left.\mathrm{R}_{1}=\mathrm{CH}_{3}\left(\mathrm{CH}_{2}\right)_{7} \mathrm{CH}=\mathrm{CH}\left(\mathrm{CH}_{2}\right)_{7}\right)$ for FA-oleate:TOPO, FA-oleate and OA:TOPO (top to bottom) over temperature range $15-75^{\circ} \mathrm{C}$. b) Shift of peak position of TOPO P (highlighted in red, $\left.\mathrm{R}_{2}=\mathrm{CH}_{3}\left(\mathrm{CH}_{2}\right)_{7}\right)$ in ${ }^{31} \mathrm{P}$ NMR for TOPO, OA:TOPO and FA-oleate:TOPO over temperature range $15-75^{\circ} \mathrm{C}$. Data in b) for TOPO and OA:TOPO is reproduced from with permission from ACS,$^{1}$ the article can be found here: https://pubs.acs.org/doi/10.1021/jacs. 8 b08978 and any further permissions related to this material should be directed to ACS. 
a)<smiles>[Z11][R6](=O)[R]([Y12])([H])OC([R])=O</smiles>

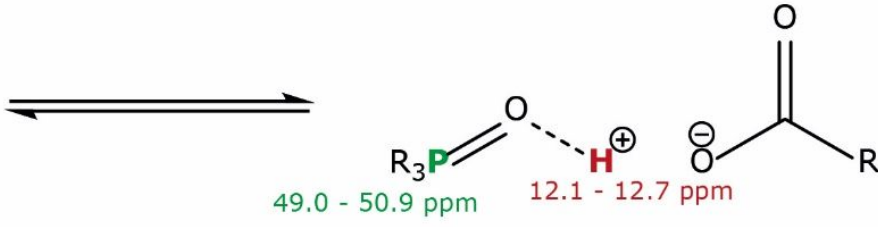

b)

$$
\mathrm{R}_{3} \mathrm{P}=\mathrm{O}
$$

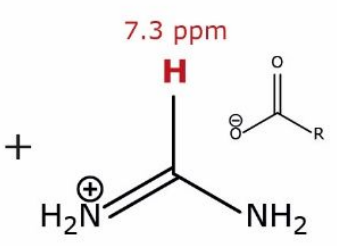

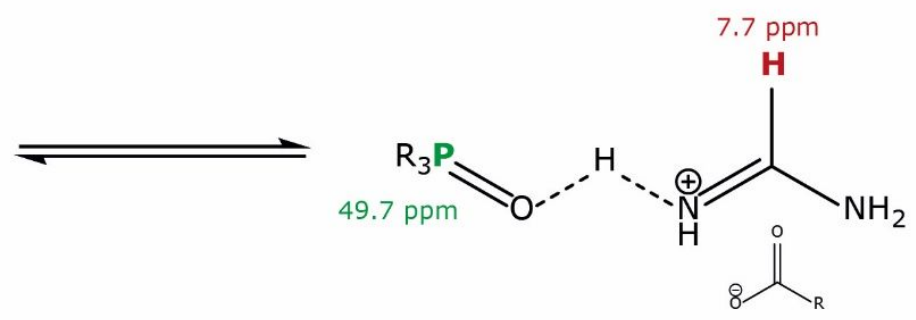

Figure S5 Proposed chemical equilibria for hydrogen bonding present in NMR solutions. a) TOPO $\left(\mathrm{R}_{3} \mathrm{P}=\mathrm{O}\right)$ and $\mathrm{OA}$ $(\mathrm{HOC}(\mathrm{O}) \mathrm{R})$ form a hydrogen bond, with the ${ }^{31} \mathrm{P}$ (green) and ${ }^{1} \mathrm{H}(\mathrm{red})$ chemical shifts of highlighted nuclei annotated. b) Additionally, TOPO $\left(\mathrm{R}_{3} \mathrm{P}=\mathrm{O}\right)$ and $\mathrm{FA}$-oleate $\left(\mathrm{HC}\left(=\mathrm{NH}_{2}{ }^{+}\right) \mathrm{NH}_{2}\right.$. $\left.{ }^{-} \mathrm{OC}(\mathrm{O}) \mathrm{R}\right)$ form a hydrogen bond between the amidinium proton and the phosphine oxide, with the ${ }^{31} \mathrm{P}$ (green) and ${ }^{1} \mathrm{H}$ (red) chemical shifts of highlighted nuclei annotated.

\section{Further Discussion}

First consider the acidic proton on OA. In Figure S4a we observe for OA:TOPO (red) a upfield shift and narrowing, as the acidic proton goes back on to OA. This is due to the neutral form of TOPO and OA forming and is in-line with ${ }^{31} \mathrm{P}$ NMR observations in Figure S4b. For FA-oleate alone (Figure S4a, black), there is significant sharpening and upfield shift across the temperature range. At higher temperatures the acidic proton will be exchanging between OA molecules faster due to a higher energy, and the molecules will be tumbling quicker. This results in a more defined position of the acidic proton on the NMR timescale, and thus a sharper peak. When TOPO is added, the same trend is observed, as shown in FA-oleate:TOPO (green) in Figure S4a.

The lack of difference in these trends could be due to TOPO preferentially interacting with FA over OA. In that case, the acidic proton on OA is free to continue to exchange between different OA molecules, as it was in the FA-oleate case. If there was a more favourable interaction between OA and TOPO in the FA-oleate:TOPO sample, then a broader peak for the acidic proton would be expected, reflecting the OA:TOPO character.

Variable temperature ${ }^{31} \mathrm{P}$ NMR is presented in Figure S4b. As seen previously, over increasing temperature there is an upfield shift in the position of neat TOPO (black squares). On the addition of OA (red triangles) this shift is intensified and attributed to the acid-base interaction of OA and TOPO. ${ }^{1}$ When FA-oleate:TOPO (green circles) is evaluated over this temperature range, the relative shift sits between that of neat TOPO and OA:TOPO. This confirms that the FA is impacting the acid-base interaction of OA and TOPO.

In Figure S5 we suggest the chemical equilibria in the NMR solutions. Firstly we show the nature of the hydrogen bonding between TOPO and the acidic proton on OA in Figure S5a, with a range of chemical shifts obtained in both ${ }^{31} \mathrm{P}$ and ${ }^{1} \mathrm{H}$ NMR depending on the concentration and presence of either Cs-oleate or FA-oleate. Where TOPO and FA-oleate are both in solution, there is another set of hydrogen bonding, this time between the amidinium proton and TOPO, Figure S5b. This bonding induces a shift in the FA methine proton by $0.4 \mathrm{ppm}$. 


\section{Synthetic Optimisation Characterisation Data}
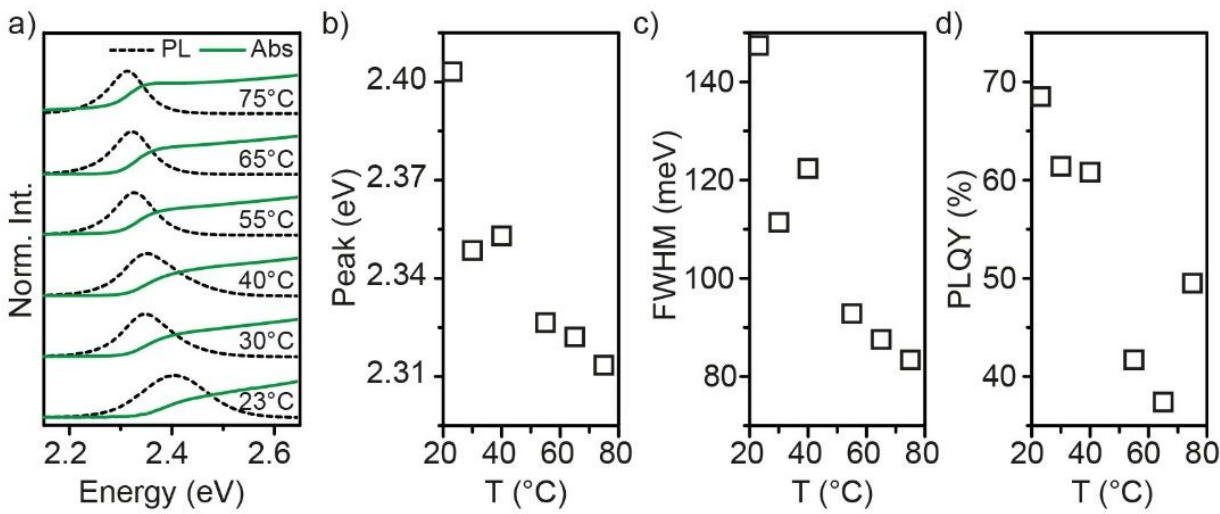

Figure S6 a) PL (black dashed) and absorption (green solid) spectra of $\mathrm{FAPbBr}_{3}$ synthesised at temperatures from 23 to 75 ${ }^{\circ} \mathrm{C}$, with the corresponding b) peak position, c) FWHM and d) PLQY

a)

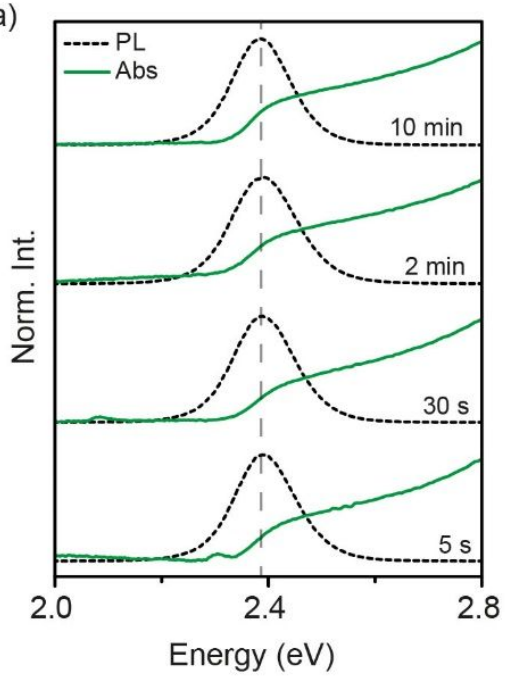

b)

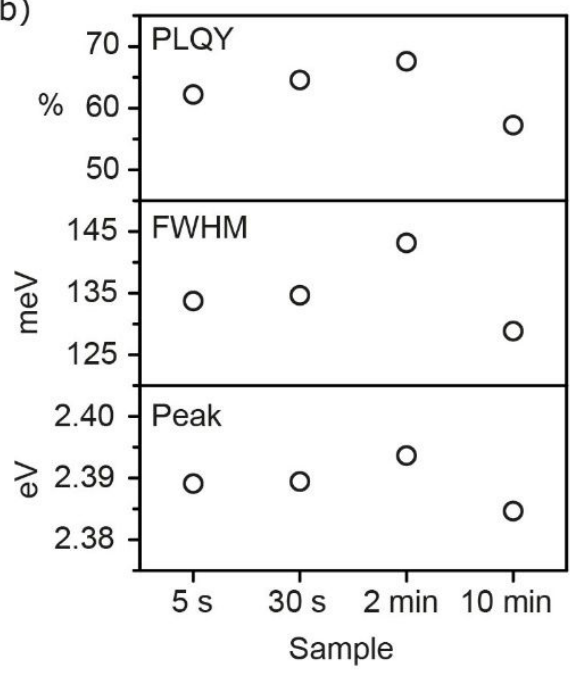

Figure S7 a) PL (black dashed) and absorption (green solid) spectra of $\mathrm{FAPbBr}_{3}$ synthesised at room temperature with growth times from 5 seconds to 10 minutes, with the corresponding b) peak position, FWHM, and PLQY (bottom to top) 

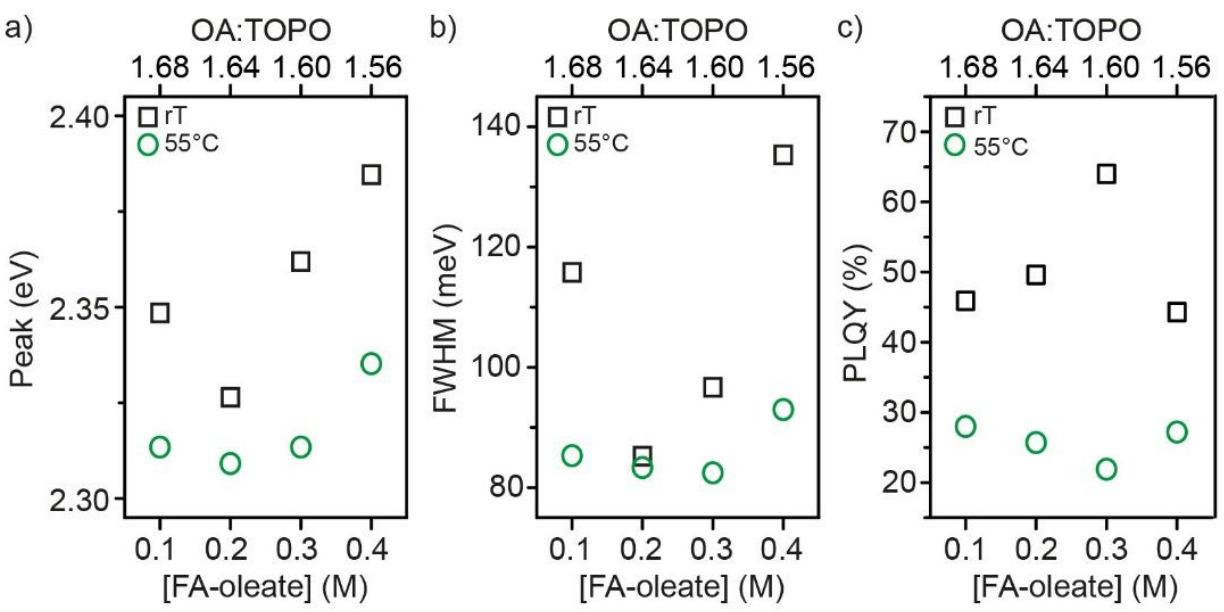

Figure S8 a) PL peak position, b) FWHM and c) PLQY of FAPbBr $r_{3}$ synthesised at $55{ }^{\circ} \mathrm{C}$ (green circle) and room temperature (black square) with varying concentrations of FA-oleate injected. Bottom x-axis denotes the concentration of FA-oleate used, and the top $\mathrm{x}$-axis is the corresponding OA:TOPO ratio, where $\mathrm{TOPO}=1$

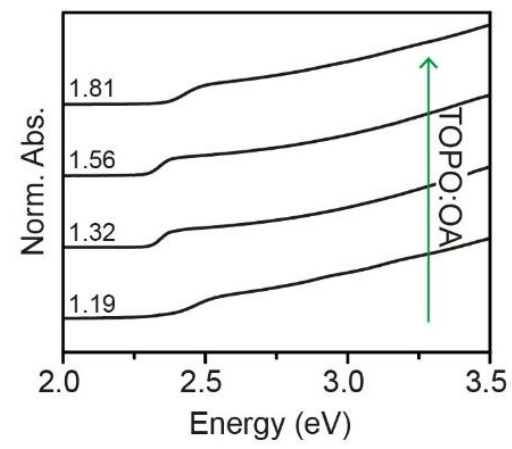

Figure S9 Absorption spectra of $\mathrm{FAPbBr}_{3}$ synthesised at room temperature with varying ratio of OA:TOPO, taken after XRD had been conducted on the pellet by washing the pellet off the quartz disc. Desired ratios are achieved by fixing either the TOPO or OA content

\section{Further Discussion}

The presence of features below $2.75 \mathrm{eV}$ in the absorption spectra of unwashed nanoparticles samples suggest that either a $0 \mathrm{D}\left(\mathrm{FA}_{4} \mathrm{PbBr}_{6}\right)$ or (quasi-)2D perovskite structure have formed. In the case of a $0 \mathrm{D}$ perovskite, as reported for $\mathrm{Cs}_{4} \mathrm{PbBr}_{6}$, hexagonal particles form and there are clear changes in the XRD pattern. ${ }^{2-5}$ Although we do observe hexagonal particles under certain reaction conditions (Figure $4 \mathrm{a} \&$ b, main text), no additional peaks are present in the XRD (Figure 4c, main text). Additionally, research published on a composite perovskite formed of $\mathrm{FA}_{4} \mathrm{PbBr}_{6}$ and $\mathrm{FAPbBr}_{3}$ assign a peak at $325 \mathrm{~nm}(3.82$ $\mathrm{eV}$ ) in the absorption spectra to the $0 \mathrm{D}$ perovskite ${ }^{6}$ Here, our features in the absorption spectra are between $2.75-3.25 \mathrm{eV}$ and so do not align with that expected for $\mathrm{FA}_{4} \mathrm{PbBr}_{6}$.

If a quasi-2D perovskite had formed, then features in the absorption spectra would be expected at given wavelengths for the layers formed. The intensity of these features would indicate the amount of the quasi-2D perovskite present in the case of a blend, or mixed particles, which has been shown for example for butylammonium-methylammonium blends. ${ }^{7}$ This was also shown in the case of $\mathrm{FA}_{2} \mathrm{PbBr}_{4}$ forming, with a strong peak in the absorption spectra at $400 \mathrm{~nm}(3.10 \mathrm{eV}){ }^{8}$ In the nanoparticles reported here, however, the features below $2.75 \mathrm{eV}$ in the absorption spectra are not at a fixed position and shift slightly with the OA:TOPO ratio. Furthermore, the most prominent peak is at $3.17 \mathrm{eV}$ (Figure $3 \mathrm{~d}$, main text) and thus does not align with previous reports of formamidinium based or blended quasi-2D perovskites. In summary, we do expect that these features arise from some additional phases but cannot identify or correlate their precise origin. 
125

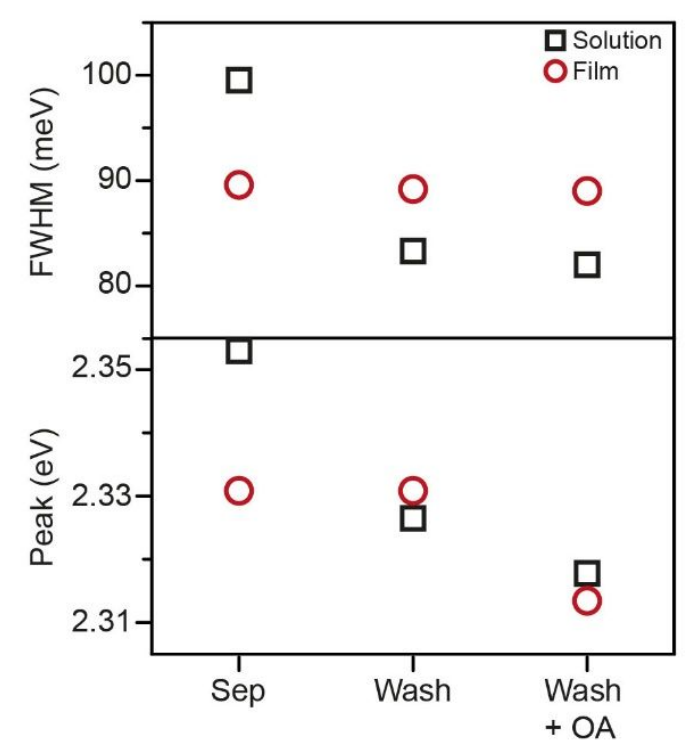

Figure S10 PL peak position (bottom) and FWHM (top) of $\mathrm{FAPbBr}_{3}$ nanoparticles washed in different ways with toluene and acetonitrile (Sep, Wash, Wash $+\mathrm{OA}$ ) in solution (black square) and drop cast films (red circle)

a)

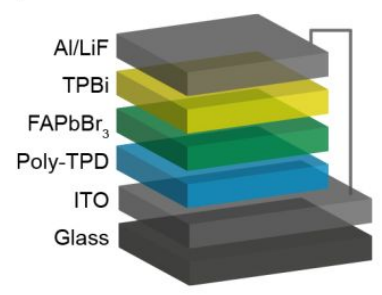

d)

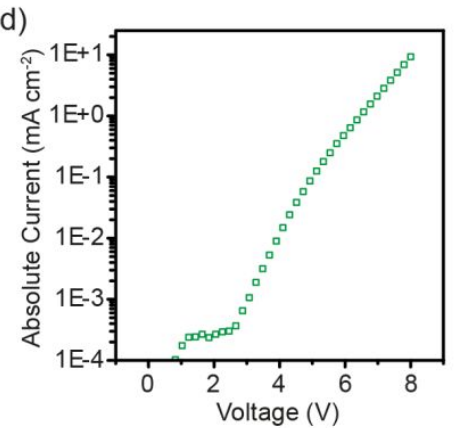

b)

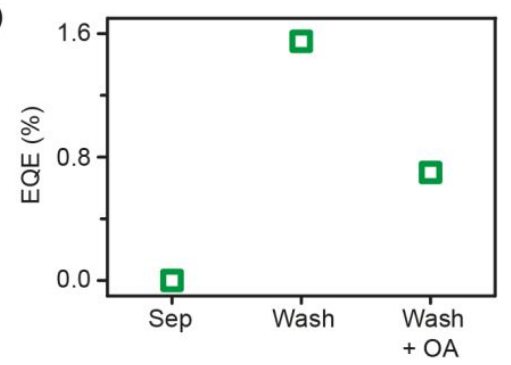

c)

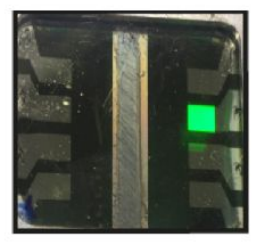

e)
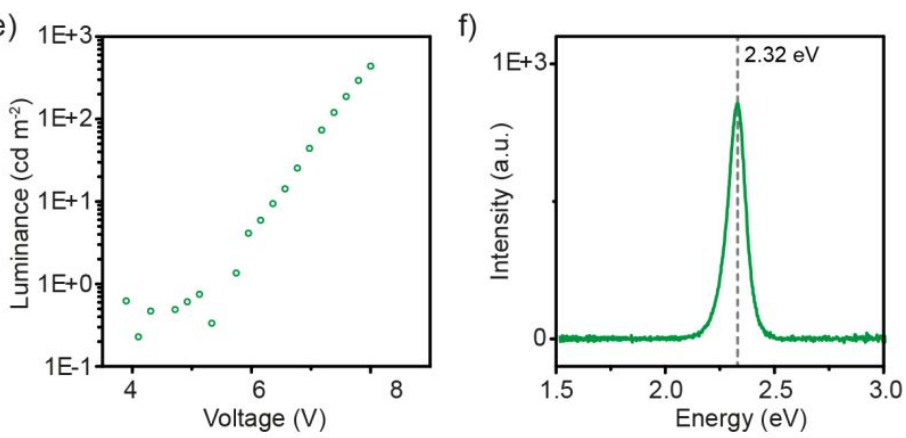

Figure S11 LED data for $\mathrm{FAPbBr}_{3}$ nanoparticle device. a) Device stack, b) external quantum efficiency (EQE) of devices made from washed nanoparticles and c) picture of operational device made from sample Wash. d) Voltage vs absolute current density, b) voltage vs luminance and c) electroluminescence spectrum with emission centred at $2.32 \mathrm{eV}$ with a FWHM of $93 \mathrm{meV}$ for champion Wash device pixel. 

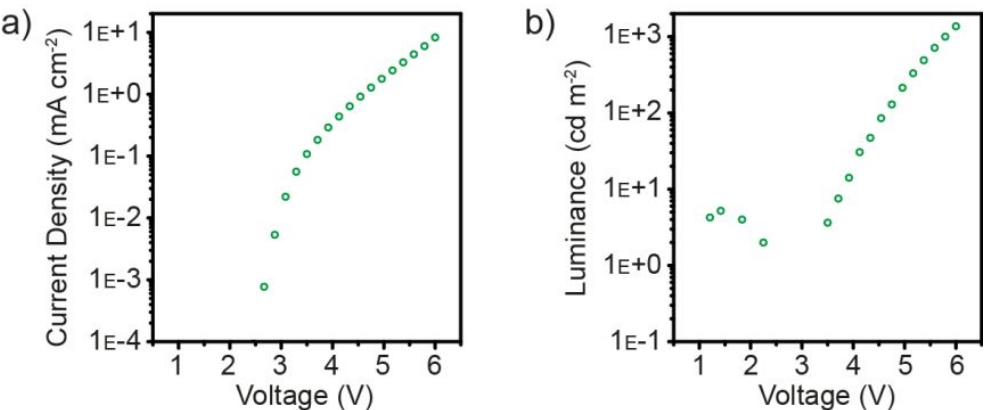

Figure S12 LED data for $\mathrm{FAPbBr}_{3}$ nanoparticle device with optimised stack. a) Voltage vs absolute current density, and b) voltage vs luminance with emission centred at $2.32 \mathrm{eV}$ with a FWHM of $90 \mathrm{meV}$ for champion Wash device pixel.
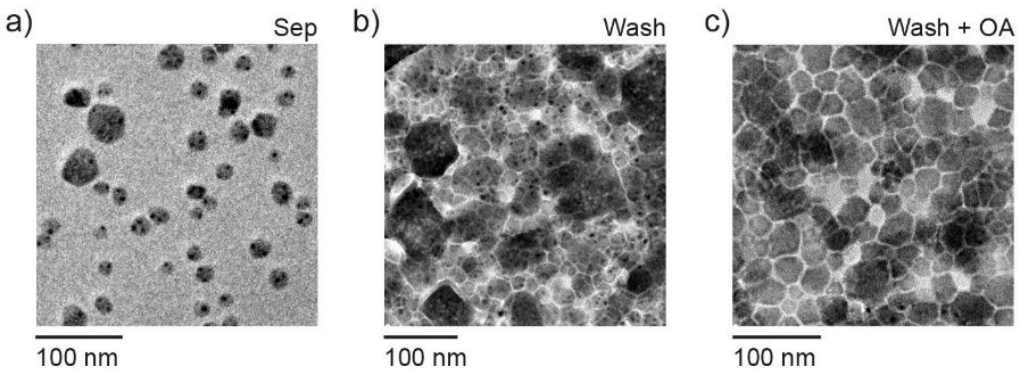

Figure S13 TEM images of $\mathrm{FAPbBr}_{3}$ nanoparticles that have been a) separated (Sep), b) washed (Wash) or c) washed with additional $\mathrm{OA}$ in solution (Wash $+\mathrm{OA}$ )

\section{References}

1. Almeida, G. et al. The Phosphine Oxide Route toward Lead Halide Perovskite Nanocrystals. Journal of the American Chemical Society 140, (2018).

2. Zhang, Y. et al. Zero-Dimensional $\mathrm{Cs}_{4} \mathrm{PbBr}_{6}$ Perovskite Nanocrystals. The Journal of Physical Chemistry Letters 8, 961-965 (2017).

3. Zhang, Y. et al. Ligand-Free Nanocrystals of Highly Emissive $\mathrm{Cs}_{4} \mathrm{PbBr}_{6}$ Perovskite. The Journal of Physical Chemistry C 122, 6493-6498 (2018).

4. Zhao, H. et al. Zero-Dimensional Perovskite Nanocrystals for Efficient Luminescent Solar Concentrators. Advanced Functional Materials 29, 1902262 (2019).

5. Quan, L. N. et al. Highly Emissive Green Perovskite Nanocrystals in a Solid State Crystalline Matrix. Advanced Materials 29, 1605945 (2017).

6. Nguyen, L. A. T. et al. Pressure-induced fluorescence enhancement of $\mathrm{FA} \alpha \mathrm{PbBr} 2+\alpha$ composite perovskites. Nanoscale 11, 5868-5873 (2019).

7. La-Placa, M.-G. et al. Photoluminescence quantum yield exceeding $80 \%$ in low dimensional perovskite thin-films via passivation control. Chem. Commun. 53, 8707-8710 (2017).

8. Shin, M. et al. Low-dimensional formamidinium lead perovskite architectures via controllable solvent intercalation. Journal of Materials Chemistry C 7, 3945-3951 (2019). 